05

\title{
Магнитно-резонансная силовая спектроскопия колебаний магнитного вихря
}

\author{
(C) В.Л. Миронов, ${ }^{1,2}$ Е.В. Скороходов, ${ }^{1}$ Д.А. Татарский, ${ }^{1,2}$ И.Ю. Пашенькин ${ }^{1}$ \\ ${ }^{1}$ Институт фризики микроструктур РАН, \\ 607680 Афронино, Нижегородская обл., Россия \\ ${ }^{2}$ Нижегородский государственный университет им. Н.И. Лобачевского, \\ 603950 Нижний Новгород, Россия \\ e-mail: mironov@ipmras.ru
}

Поступило в Редакцию 31 марта 2020 г.

В окончательной редакции 31 марта 2020 г.

Принято к публикации 31 марта 2020 г.

\begin{abstract}
Приведены результаты микромагнитного моделирования и экспериментальных исследований методом магнитно-резонансной силовой спектроскопии вынужденных колебаний намагниченности круглого диска $\mathrm{NiFe}$ во внешнем продольном магнитном поле. Основное внимание уделено низкочастотному резонансу, связанному с гиротропным движением кора магнитного вихря. Показано, что приложение внешнего магнитного поля в плоскости образца приводит к значительному сдвигу резонансной частоты гиромоды. Обсуждено влияние неоднородного магнитного поля зонда на характер колебаний намагниченности.
\end{abstract}

Ключевые слова: магнитный вихрь, гиротропная мода, магнитно-резонансная силовая микроскопия, наноосциллятор.

DOI: $10.21883 /$ JTF.2020.11.49968.105-20

\section{Введение}

В круглых ферромагнитных дисках распределение намагниченности представляет собой магнитный вихрь [1] (рис. 1). Оболочка вихря лежит в плоскости диска и имеет завихренность либо по часовой, либо против часовой стрелки. В центре вихря расположена узкая область - кор, имеющая направление намагниченности, выходящее из плоскости диска. Низшая частота колебаний такой системы соответствует гиротропной моде, в

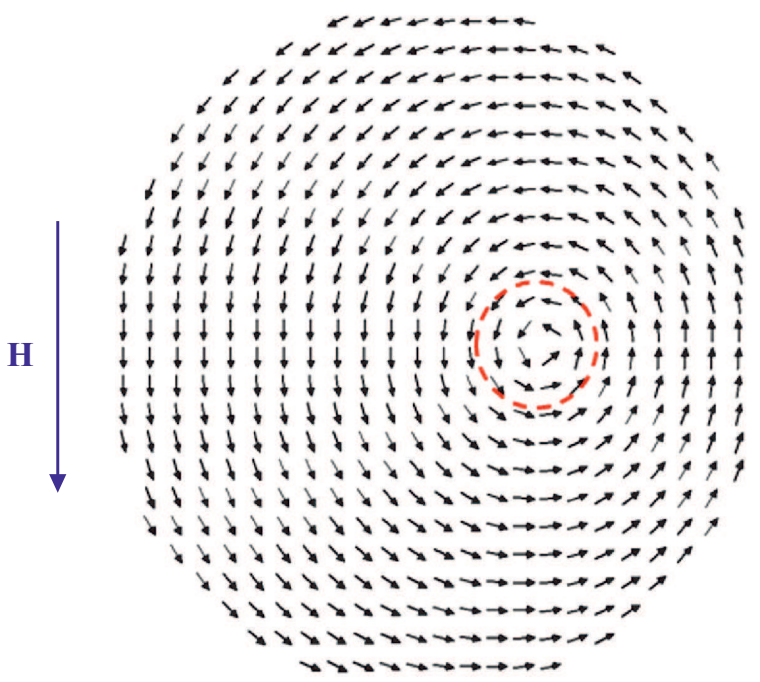

Рис. 1. Модельное распределение намагниченности диска в виде магнитного вихря во внешнем магнитном поле. Пунктиром схематически показано движение центра вихря, соответствующее низкочастотному резонансу — гиротропной моде. которой центр вихря движется по окружности вокруг состояния равновесия. В целом интерес к исследованию гиротропной моды связан, прежде всего, с возможностью изменения ее частоты под действием внешнего магнитного поля, а следовательно, и возможным применением вихревых дисков для разработки перестраиваемых по частоте наноосцилляторов или субмикронных детекторов поля [2,3].

Низкочастотный резонанс, связанный с гиротропным движением кора магнитного вихря в ферромагнитном диске, был предсказан теоретически $[4,5]$ и измерен с помощью полярного эффекта Керра [6] и рентгеновской фотоэмиссионной электронной микроскопии $[7,8]$. Также были проведены измерения резонансов в массиве дисков, высаженных на сигнальном электроде копланарной линии $[9,10]$, и в одиночных дисках методом магнитнорезонансной силовой микроскопии (МРСМ) [11]. В данных работах были исследованы зависимости резонансной частоты гиротропной моды движения кора вихря от аспектного соотношения диска [6-8] и от величины внешнего магнитного поля, приложенного перпендикулярно плоскости [10-12]. В настоящей работе мы использовали метод МРСМ для исследования зависимости резонансных колебаний кора вихря в круглых пермаллоевых дисках от внешнего магнитного поля, приложенного в плоскости образца.

\section{1. Эксперимент}

В эксперименте исследовались круглые диски из пермаллоя $\left(\mathrm{Ni}_{80} \mathrm{Fe}_{20}\right)$, изготовленные методом взрывной 
(„lift-off“) литографии. Вначале на стеклянной подложке (толщиной $170 \mu \mathrm{m})$ выращивался слой пермаллоя толщиной $60 \mathrm{~nm}$. Затем сверху наносился слой (толщиной $150 \mathrm{~nm}$ ) позитивного электронного резиста полиметилметакрилата (ПММА 495 С2) методом центрифугирования. Начальная маска в виде массива кругов диаметром $1 \mu \mathrm{m}$ формировалась в резисте посредством экспонирования электронным пучком в микроскопе SUPRA 50VP с литографической приставкой ELPHY PLUS (компания „CarlZeiss“, Jena). Затем облученные области резиста, соответствующие будущим дискам, удалялись в органическом растворителе. После этого методом магнетронного напыления на образец наносился тонкий (толщиной $30 \mathrm{~nm}$ ) слой ванадия. Затем была проведена операция „lift-off“ в ацетоне с применением ультразвука, в результате чего были сформированы диски ванадия на пермаллое, которые служили защитной маской при ионном $\mathrm{Ar}^{+}$травлении образца. В итоге был сформирован массив дисков диаметром $1 \mu \mathrm{m}$ и толщиной $60 \mathrm{~nm}$, упорядоченный на квадратной решетке с периодом $15 \mu \mathrm{m}$. Процедура изготовления образцов с использованием ионного травления позволяет получить структуры с более совершенными границами, чем при обычной процедуре „lift-off‘ что позволяет лучше управлять магнитными свойствами полученных образцов. Для минимизации электростатических эффектов образец дополнительно покрывался тонкой (толщиной $5 \mathrm{~nm}$ ) пленкой платины.

Вынужденные колебания намагниченности дисков исследовались с помощью магнитно резонансного силового микроскопа (МРСМ), созданного в ИФМ РАН на базе сканирующего зондового микроскопа „Solver HV“ [13-15]. Данная установка позволяет проводить исследования спектров колебаний намагниченности в диапазоне частот $0.01-20 \mathrm{GHz}$ и в магнитных полях до $3 \mathrm{kOe}$. В качестве зондового датчика использовался специальный мягкий кантилевер с резонансной частотой $6.7 \mathrm{kHz}$ и жесткостью $0.03 \mathrm{~N} / \mathrm{m}$, на который наклеивалась частица $\mathrm{SmCo}$ размером $\sim 10 \mu \mathrm{m}$. Для СВЧ накачки образцов использовался перестраиваемый генератор „SPS-20“ (компания „Спектран“, г. Саратов). Мощность накачки составляла $-2 \mathrm{dBm}(0.63 \mathrm{~mW})$. Образец располагался на планарной закороченной полосковой линии в пучности магнитного СВЧ поля, направленного в плоскости диска. Внешнее подмагничивающее поле $H$ создавалось с помощью электромагнита постоянного тока и было направлено в плоскости образца перпендикулярно переменному полю. Измерения проводились в вакууме $(0.1 \mathrm{~Pa})$, при этом добротность механических колебаний кантилевера составляла 950.

\section{2. Моделирование}

Микромагнитное моделирование динамики намагниченности диска проводилось на основе численного интегрирования уравнения Ландау-Лифшица для магнитного момента с использованием симулятора MuMax3 [16]. Ферромагнитный резонанс рассчитывался в цилиндрическом диске диаметром $1 \mu \mathrm{m}$ и толщиной $60 \mathrm{~nm}$. Нами были использованы следующие материальные параметры: намагниченность насыщения $M_{s}=800 \mathrm{kA} / \mathrm{m}$, обменная константа $J=13 \mathrm{pJ} / \mathrm{m}$, параметр затухания $\alpha=0.015$ [17]. Расчеты проводились на прямоугольной сетке, содержащей $256 \times 256 \times 1$ узлов, шаг сетки в плоскости составлял $4 \mathrm{~nm}$. Сетка в плоскости выбрана таким образом, чтобы ее шаг был меньше обменной длины $L_{\mathrm{ex}} \approx 10 \mathrm{~nm}$. Дополнительное моделирование показало, что увеличение числа узлов по толщине диска не приводит к изменению спектров и для получения корректных результатов достаточно считать намагниченность однородной по толщине. Для определения резонансной частоты колебаний вихря на систему подавался кратковременный (длительностью $0.1 \mathrm{~ns}$ ) импульс магнитного поля амплитудой $1 \mathrm{Oe}$, направленного в плоскости диска. Затем записывалась осциллограмма (100-150 периодов) релаксационных колебаний среднего значения намагниченности. Низшая резонансная частота колебаний, соответствующая гиротропной моде, выделялась посредством быстрого преобразования Фурье. В численном эксперименте исследовался сдвиг резонансной частоты от внешнего поля.

\section{3. Результаты и обсуждение}

В МРСМ экспериментах снимались зависимости амплитуды колебаний кантилевера от частоты излучения СВЧ накачки во внешнем продольном подмагничивающем поле. Зондовый датчик располагался над центральной частью диска. Расстояние зонд-образец составляло $3 \mu \mathrm{m}$. Частота накачки перестраивалась в диапазоне 0.1-2 GHz. На рис. 2 приведены МРСМ спектры диска в различных магнитных полях. Резонансу соответствуют провалы на зависимостях амплитуды колебаний кантилевера от частоты. Из рисунка видно, что ширина резонансных кривых более $150 \mathrm{MHz}$, что может быть связано с неоднородностью поля зонда.

Зависимость резонансной частоты гиротропной моды от величины внешнего магнитного поля приведена на рис. 3 (кривая 1). Видно, что в данной области полей зависимость линейная.

Микромагнитное моделирование показало, что в нулевом внешнем продольном поле расчетная $(\sim 450 \mathrm{MHz})$ и экспериментальная $(\sim 620 \mathrm{MHz})$ частоты гиротропной моды не совпадают. Известно, что приложение внешнего поля вдоль оси $z$ параллельно намагниченности кора вихря увеличивает собственную частоту гиротропной моды [18]. Источником такого поля в МРСМ эксперименте является частица $\mathrm{SmCo}$, расположенная на конце кантилевера $[19,20]$. Для учета поля МРСМ зонда мы использовали простейшую модель, в которой поле зонда рассчитывалось как эффективное поле магнитного 


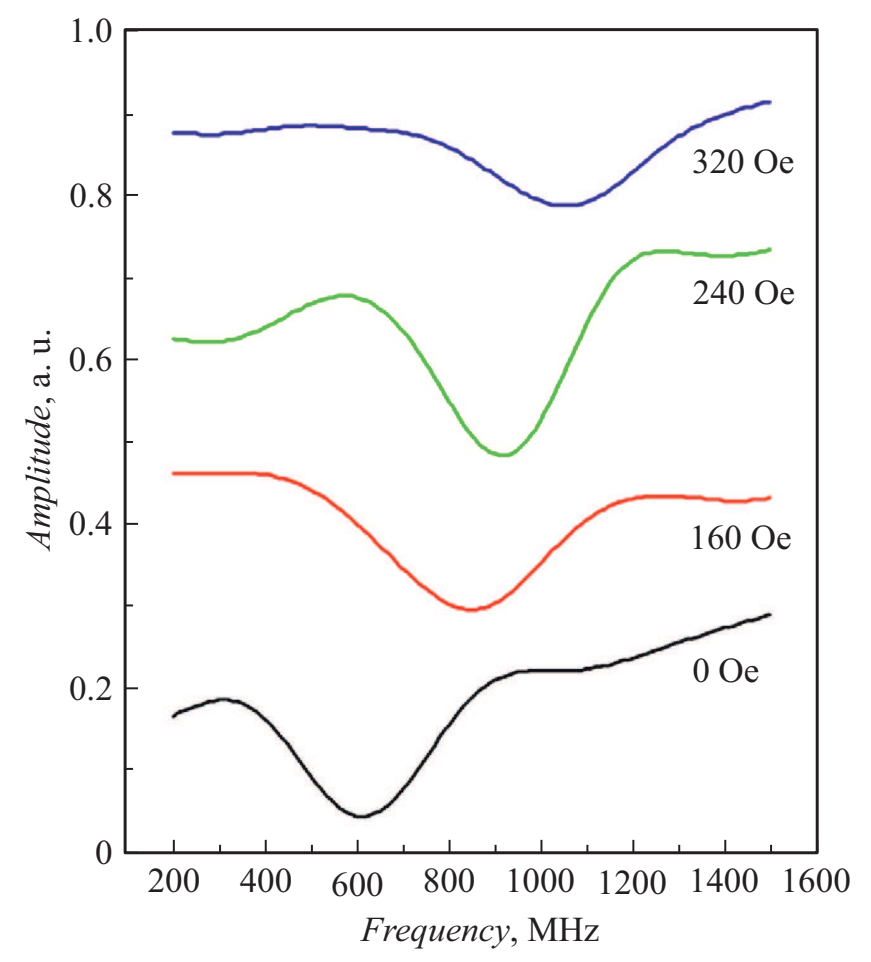

Рис. 2. Экспериментальные МРСМ спектры, соответствующие гиротропной моде колебаний магнитного вихря во внешнем магнитном поле. Спектры сдвинуты в вертикальном направлении для лучшего восприятия.

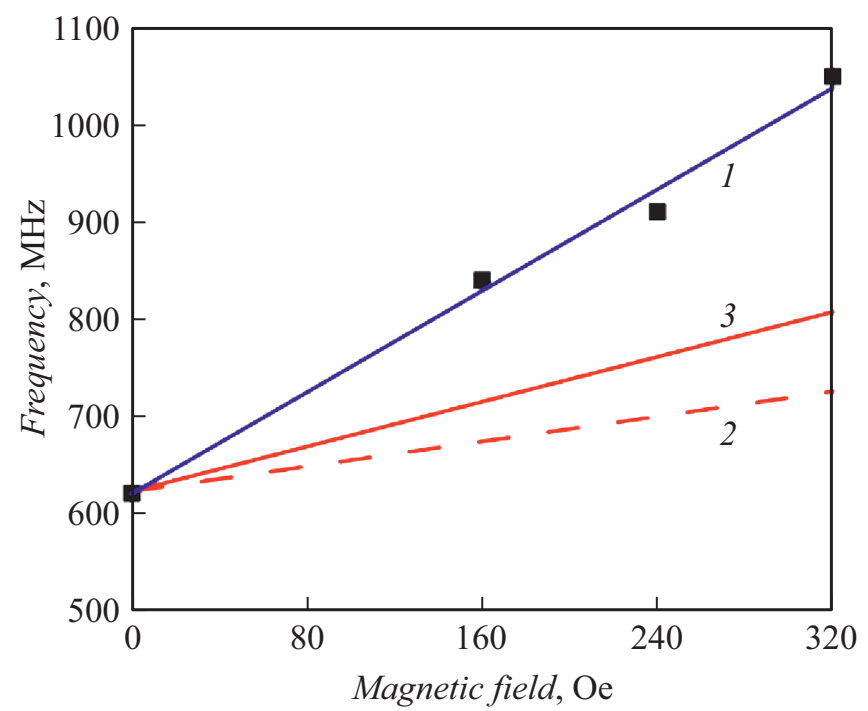

Рис. 3. Зависимости резонансной частоты гиротропной моды от продольного магнитного поля: 1 - экспериментальная зависимость (квадратами показаны экспериментально измеренные значения резонансной частоты); 2 - модельная зависимость с учетом поля зонда, но без учета изменения его высоты; 3 модельная зависимость с учетом поля зонда и изменения его высоты.

монополя, расположенного над диском [21]. Расстояние между монополем и диском выбиралось в соот- ветствие с экспериментальным значением расстояния зонд-образец, заряд монополя служил подгоночным параметром.

Приложение внешнего продольного поля приводит к сдвигу кора вихря относительно центра диска, что сопровождается изменением профиля потенциальной энергии для вихря внутри диска и приводит к увеличению резонансной частоты гиротропной моды [22]. При этом кор сдвигается не перпендикулярно внешнему полю из-за наличия радиальной компоненты поля зонда (рис. 4). Микромагнитное моделирование показало, что такого элементарного учета поля зонда недостаточно для объяснения экспериментально наблюдаемого сдвига резонансной частоты (рис. 3 , кривая 2). Дополнительный сдвиг связан с изменением высоты зонда из-за действия силы на частицу SmCo со стороны внешнего поля. Зависимость резонансной частоты в этом случае приведена на рис. 3 (кривая 3). Высота зонда при расчетах изменялась с $3.5 \mu \mathrm{m}$ при $H=0$ Ое до $2.8 \mu \mathrm{m}$ для внешнего поля $H=320$ Ое. Отметим, что мы использовали наиболее простую модель системы зонд-образец-внешнее поле. В используемой нами модели не учитывались такие факторы как наклон магнитного зонда по отношению к плоскости образца, смещение зонда относительно центра диска, а также возможное более сложное распределение поля зонда вследствие сложной формы, наклона магнитного момента и неоднородной намагниченности $\mathrm{SmCo} \mathrm{частицы.}$
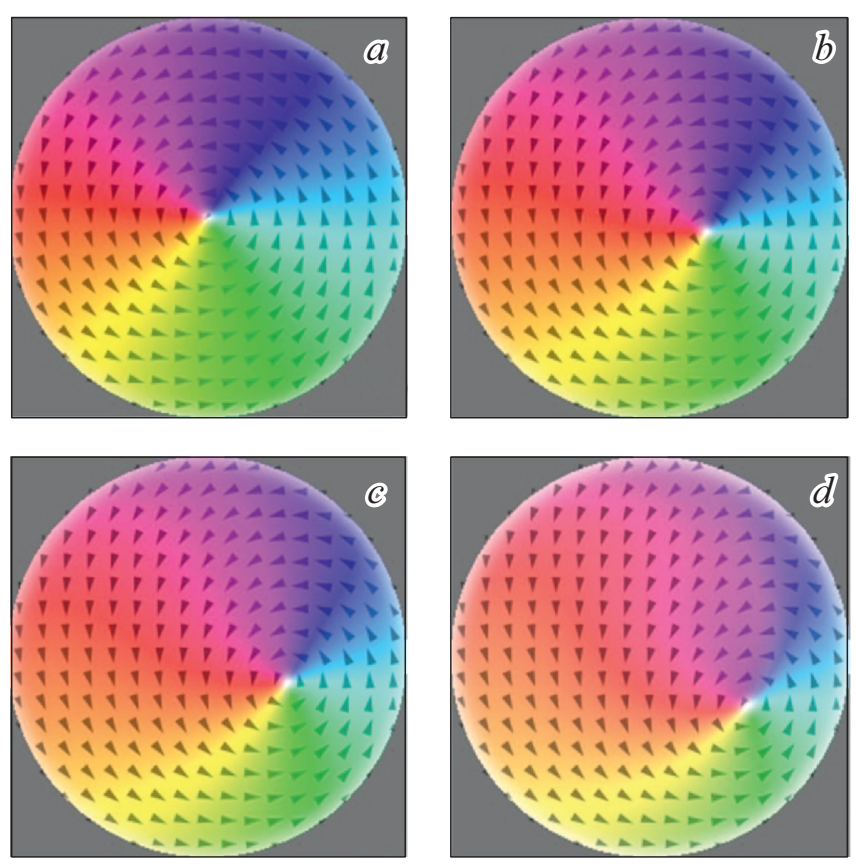

Рис. 4. Модельные распределения намагниченности для вихря в однородном продольном поле с учетом влияния поля $H$ и высоты зонда $d$. Поле прикладывается вертикально, сверху вниз: $a-H=0 \mathrm{Oe}, d=3.5 \mu \mathrm{m} ; b-H=160 \mathrm{Oe}, d=3.3 \mu \mathrm{m}$; $c-H=240 \mathrm{Oe}, d=3.1 \mu \mathrm{m} ; d-H=320 \mathrm{Oe}, d=2.8 \mu \mathrm{m}$. 


\section{Заключение}

Таким образом, были проведены МРСМ исследования гиротропной моды колебаний магнитного вихря в одиночном диске диаметром $1 \mu \mathrm{m}$ и толщиной $60 \mathrm{~nm}$ в продольном внешнем магнитном поле. Показано, что зависимость резонансной частоты данной моды от внешнего магнитного поля имеет линейный характер в диапазоне полей 0-300 Ое. Сравнение экспериментальных результатов и результатов микромагнитного моделирования показало, что на резонансные свойства вихря большое влияние оказывает неоднородного поля МРСМ зонда. В дальнейшем планируется изучение особенностей колебаний вихря в сильно неоднородном магнитном поле и построение более реалистичной модели взаимодействия зонда с образцом.

\section{Благодарности}

Авторы выражают благодарность Р.В. Гореву за помощь в моделировании и В.В. Рогову за помощь в приготовлении образца.

\section{Финансирование работы}

Работа выполнена при поддержке РФФИ (проект № 18-02-00247) и в рамках государственного здания (контракт № 0035-2019-0022-С-01). В экспериментах использовалось оборудование центра коллективного пользования „Физика и технология микро- и наноструктур“ ИФМ РАН.

\section{Конфликт интересов}

Авторы заявляют, что у них нет конфликта интересов.

\section{Список литературы}

[1] Lebrun R., Locatelli N., Tsunegi S., Grollier J., Cros V., Abreu Araujo F., Kubota H., Yakushiji K., Fukushima A., Yuasa S. // Phys. Rev. App. 2014. Vol. 2. P. 061001-1-061001-6. https://doi.org/10.1103/PhysRevApplied.2.061001

[2] Braganca P.M., Gurney B.A., Wilson B.A., Katine J.A., Maat S., Childress J.R. // Nanotechnology. 2010. Vol. 21. P. 235202-1-235202-6. https://doi.org/10.1088/0957-4484/21/23/235202

[3] Metlov K.L., Lee Y. // Appl. Phys. Lett. 2008. Vol. 92. P. 112506-1-112506-3. https://doi.org/10.1063/1.2898888

[4] Usov N.A., Kurkina L.G. // J. Magn. Magn. Mater. 2002. Vol. 242. P. 1005-1008. https://doi.org/10.1016/S0304-8853(01)01363-4

[5] Guslienko K.Yu., Ivanov B.A., Novosad V., Otani Y., Shima H., Fukamichi K. // J. Appl. Phys. 2002. Vol. 91. P. 8037-8039. https://doi.org/10.1063/1.1450816

[6] Park J.P., Eames P., Engebretson D.M., Berezovsky J., Crowell P.A. // Phys. Rev. B. 2003. Vol. 67. P. 020403-1-020403-4. https://doi.org/10.1103/PhysRevB.67.020403
[7] Guslienko K.Yu., Han X.F., Keavney D.J., Divan R., Bader S.D. // Phys. Rev. Lett. 2006. Vol. 96. P. 067205-1067205-4. https://doi.org/10.1103/PhysRevLett.96.067205

[8] Choe S.-B., Acremann Y., Scholl A., Bauer A., Doran A., Stohr J., Padmore H.A. // Science. 2004. Vol. 304. P. 420 422. https://doi.org/10.1126/science. 1095068

[9] Novosad V., Fradin F., Roy P., Buchanan K., Guslienko K.Y., Bader S.D. // Phys. Rev. B. 2005. Vol. 72. P. 024455-1024455-5. https://doi.org/10.1103/PhysRevB.72.024455

[10] Ким П.Д., Орлов В.А., Прокопенко В.С., Замай С.С., Прини В.Я., Руденко Р.Ю., Руденко Т.В. // ФТТ. 2015. Т. 57. Вып. 1. C. 29-36. [Kim P.D., Orlov V.A., Prokopenko V.S., Zamaic S.S., Prints V.Ya., Rudenko R.Yu., Rudenko T.V. // Phys. Sol. State. 2015. Vol. 57. P. 30-37.]

[11] Pigeau B., de Loubens G., Klein O., Riegler A., Lochner F., Schmidt G., Molenkamp L.W., Tiberkevich V.S., Slavin A.N. // Appl. Phys. Lett. 2010. Vol. 96. P. 132506-1-132506-3. https://doi.org/10.1063/1.3373833

[12] Guslienko K.Yu. // Appl. Phys. Lett. 2006. Vol. 89. P. 0225101-022510-2. https://doi.org/10.1063/1.2221904

[13] Скороходов Е.В., Сапожсников М.В., Резник А.Н., Поляков В.В., Быков В.А., Володин А.П., Миронов В.Л. // Приборы и техника эксперимента. 2018. № 5. С. 140-145.

[14] Скороходов Е.В., Сапожников М.В., Горев Р.В., Володин А.П., Миронов В.Л. // ФТТ. 2018. Т. 60. Вып. 11. C. $2213-2218$.

https://doi.org/10.21883/FTT.2018.11.46665.25NN

[Skorokhodov E.V., Sapozhnikov M.V., Gorev R.V., Volodin A.P., Mironov V.L. // Phys. Sol. State. 2015. Vol. 60. N 11. P. 2254-2258. https://doi.org/10.1134/S1063783418110306]

[15] Горев Р.В., Скороходов Е.В., Миронов В.Л. // ЖТФ. 2019. Т. 89. Вып. 11. С. 1646-1649. https://doi.org/10.21883/JTF.2019.11.48322.126-19 [Gorev R.V., Skorokhodov E.V., Mironov V.L. // Tech. Phys. 2019. Vol. 64. P. $1556-1559$. https://doi.org/10.1134/S1063784219110112]

[16] Vansteenkiste A., Leliaert J., Dvornik M., Helsen M., GarciaSanchez F., Van Waeyenberg B. // AIP Advances. 2014. Vol. 4. P. 107133-1-107133-22. https://doi.org/10.1063/1.4899186

[17] Liu Y., Jia M., Li H., An Du // J. Magn. Magn. Mater. 2016. Vol. 401. P. 806-811. https://doi.org/10.1016/j.jmmm.2015.10.136

[18] Fried J.P., Metaxas P.J. // Phys. Rev. B. 2016. Vol. 93. P. 064422-1-064422-8. https://doi.org/10.1103/PhysRevB.93.064422

[19] Mironov V.L., Fraerman A.A., Gribkov B.A., Ermolayeva O.L., Klimov A.Yu., Gusev S.A., Nefedov I.M., Shereshevskii I.A. // Phys. Met. Metall. 2010. Vol. 110. N 7. P. 708-734.]

[20] Миронов В.Л., Ермолаева О.Л. // Поверхность. Рентгеновские, синхротронные и нейтронные исследования. 2007. № 8. C. 37-41. [Mironov V.L., Yermolaeva O.L. // J. Surf. Investigation. X-ray, Synchrotron and Neutron Techniq. 2007. Vol. 1. P. 466-470.]

[21] Lohau J., Kirsch S., Carl A., Dumpich G., Wassermann E.F. // J. Appl. Phys. 1999. Vol. 86. P. 3410-3417. https://doi.org/10.1063/1.371222

[22] Buchanan K.S., Roy P.E., Grimsditch M., Fradin F.Y., Guslienko K.Yu., Bader S.D., Novosad V. // Phys. Rev. B. 2006. Vol. 74. P. 064404-1-064404-5. https://doi.org/10.1103/PhysRevB.74.064404 